\title{
Reconstruction of High Resolution Computed Tomography Image from Sinogram Space Using Adaptive Row Projection
}

\author{
Osama A. Omer \\ Department of Electrical Engineering, Aswan Faculty of Engineering, \\ Aswan University, Aswan 81542, Egypt \\ omer.osama@aswu.edu.eg
}

\begin{abstract}
We deal with the reconstruction of the high-resolution (HR) computed tomography (CT) image from the CT projection data (Sinogram). Spatial resolution is one of the important parameters of CT images. Spatial resolution is a measure of how close to each other two objects that can still be distinguished. The spatial resolution of CT images depends on the field of view which in turn depends on the number of projections and the number of samples per projection. One way to increase the spatial resolution of the reconstructed images is to reduce the pixel size; however, this may require hardware alteration. Another way to increase the spatial resolution is to apply super-resolution (SR) technique on the lowresolution (LR) images. The conventional method for resolution enhancement is to apply SR technique as a post-process after LR CT images reconstruction. One drawback of this conventional method is that; performing two consequence steps, reconstruction and $S R$, requires more parameters to be tuned. Unlike the conventional method, we propose to simultaneously estimate HR image with the reconstruction step from the CT projection data, which indeed reduce the overall processing time and the required tuned parameters. On the other hand, even if the back-projection (BP) method is attractive because of its simplicity and low computational cost, it produces sub-optimal images with respect to artifacts, resolution, and noise. On contrast, iterative image reconstruction allows to easily model constraints and to incorporate prior knowledge which leads to better image quality. Therefore, in this paper, we employ the iterative reconstruction based on regularized Kaczmarz minimization algorithm, for its fast convergence, in the resolution enhancement as a post-process as well as simultaneous reconstruction and resolution enhancement methods.
\end{abstract}

Keywords: CT reconstruction, resolution enhancement, Sinogram

\section{Introduction}

Computed tomography (CT) is a medical imaging procedure that uses computer-processed $\mathrm{X}$-rays to produce tomographic images of specific parts of the body. This approach was first introduced by Hounsfield, who built a prototype head scanner and tested it in 1971 [1]. Since that time, the usage of CT has increased dramatically in many countries. These tomographic images are used for diagnostic purposes in various medical disciplines. The diagnostic medicine is highly affected by this technique, since it has enabled doctors to view internal organs with surprising precision and safety to the patient. 
An important part of any CT system is the reconstruction method, which transforms the measured CT projected data into images [2]. The reconstruction of CT image from the acquired data is an inverse problem. Reconstruction methods for CT can be either analytical or iterative [3]. Analytical methods can be either exact or non-exact. It is not always possible to exactly solve the inverse problem directly. Moreover, due to the high computation, exact methods are not found in clinical use. In this case, manufacturers of clinical CT systems employ non-exact methods to approximate the solution, which might cause visible reconstruction artifacts in the image. Iterative reconstruction approaches can reach the correct solution using multiple iteration steps, which allows obtaining a better reconstruction at the cost of a higher computation time [4-8]. The iterative algorithms share the main steps, namely, start with an assumed image, compute projections from the image based on the forward imaging model, compare the calculated projection data with the actual projection data and update the image based upon the difference between the calculated and the actual projections.

The main important components in iterative image reconstruction algorithms are:

1. A system model that relates the unknown object to the measurements that would be recorded in the absence of noise.

2. A cost function that is to be minimized to estimate the image coefficient.

3. An algorithm for minimizing the cost function.

Among a wide range of components in iterative image reconstruction algorithms, algorithms with linear system, regularized $\mathrm{L}_{2}$-norm cost function and Kaczmarz minimization algorithm have special concern in this paper.

On the other hand, the resolution of the reconstructed image depends, among the other things, on the field of view which is defined as the parameter that determines how much anatomy is scanned. Increasing the field of view increases the pixel size, in case of fixed matrix size, which reveals the amount of patient data each pixel contains. The resolution of CT images is limited due to the band-limited reconstruction process [9]. One way to increase the CT image resolution is to use HR CT technology that uses a narrow X-ray beam [10]; this requires hardware alteration which requires changing the conventional technology. Another way to increase the spatial resolution of the reconstructed images is to reduce the pixel size; however, this also may require hardware alteration. The other way to increase the spatial resolution is to apply SR techniques on the LR images. Digital image processing methods to obtain a HR image from LR observations have been investigated by many researchers [1114]. In [11-13], the resolution enhancement are done as a post-processing step, which increases the computational time, while the resolution enhancement in [14] is done with the reconstruction step alternatively, where the resolution enhancement is done in the Sinogram space by projecting the HR image in the Sinogram space. In [14], the quality of BP reconstruction is improved by iteratively reconstructing the CT image and super-resolve the reconstructed image. However, the BP reconstruction results in sub-optimal images. In addition, the restoration approaches have been adopted to enhance CT image resolution [9, 15-16]. Resolution enhancement algorithms are an interesting way to increase the resolution of images. They are based on the fact that, by combining various LR and highly correlated images, it is possible to obtain a HR image by using the information from different images.

The goal in this paper is to reconstruct a HR CT image using multiple measurements. Unlike the conventional approaches [11-13] that are done as a post-process; we propose to solve the resolution enhancement problem simultaneously with the reconstruction problem in the Sinogram space using iterative technique. Unlike the work done in [14], which used 
projection of HR in the Sinogram space, the proposed approach does not use analytical projection for the reconstruction step. The contributions of this paper are:

- We adopted the regularized Kaczmarz minimization algorithm in the post-processing SR algorithm.

- We proposed a simultaneous CT reconstruction and resolution enhancement algorithm.

\section{CT Reconstruction}

The CT imaging system can be modeled in matrix-vector multiplication as [1]

$\underline{U}=\mathbf{S} \underline{\mu}+\underline{\eta}$,

where $\mathbf{S}$ is the system matrix that relates the acquired CT data with the pixel values, $\underline{\mathrm{U}}$ is the measured data, $\underline{\mu}$ is the CT image and $\underline{\eta}$ is the system noise. As an inverse problem, estimating $\underline{\mu}$ can be done by minimizing [1]

$$
J(\underline{\mu})=\|\mathbf{S} \underline{\mu}-\underline{U}\|_{2}^{2}+\lambda\|\underline{\mu}\|_{2}^{2}
$$

where $\|.\|_{2}^{2}$ is the $L_{2}$-norm and $\lambda$ is the regularization parameter. The first term of (2) is the error measure term and the second term is the regularization term. It is well known [17] that the minimization of this function is equivalent to solving the regularized normal system of equations

$\underline{\widehat{U}}=\widehat{\boldsymbol{S}} \mu$

where $\widehat{\boldsymbol{S}}=\boldsymbol{S}^{*} \boldsymbol{S}+\lambda \boldsymbol{I}, \widehat{U}=\boldsymbol{S}^{*} \underline{U}, \mathbf{S}^{*}$ denotes the conjugate transpose of $\mathbf{S}$ and $\mathbf{I}$ is the identity matrix. Solving (3) can be done recursively, by using adaptive row projection $[9,18]$, as

$\mu^{j+1}=\mu^{j}+\frac{u_{k}-\left\langle\bar{s}_{k}, \mu^{j}\right\rangle_{2}}{\left\|s_{k}\right\|_{2}^{2}} \bar{s}_{k}$

where $\mathrm{j}$ denotes the inner iteration, $\mathrm{u}_{\mathrm{k}}$ is the k-th component of $\underline{\underline{U}}$ and $\bar{S}_{\mathrm{k}}$ is the conjugate complex of $\mathrm{s}_{\mathrm{k}}$ which is the k-th row of $\widehat{\boldsymbol{S}}$.

\section{Resolution Enhancement of CT Images}

\subsection{Resolution Enhancement Problem Description}

The forward LR image formation can be simply stated as [19]

$\underline{\mu}_{i}=\mathbf{D B}_{i} \mathbf{W}_{i} \underline{X}+\underline{V}_{i} \quad i=1: N$ 
Where $\mathbf{W}_{\mathrm{i}}$ is the geometric motion operator between the HR image $\mathrm{X}$ and the $\mathrm{i}$-th LR image, $\mu_{\mathrm{k}}$. The system point spread function (PSF) is modelled by the sparse matrix $\mathbf{B}_{\mathrm{i}}$, and $\mathbf{D}$ is a sparse matrix that represents the decimation operator. The vector $\underline{V}_{i}$ is the system noise and $\mathrm{N}$ is the number of available LR images. For convenience, concatenate all the measurements in one vector as follows

$$
\underline{\boldsymbol{\mu}}=\left[\begin{array}{c}
\underline{\boldsymbol{\mu}}_{1} \\
\underline{\boldsymbol{\mu}}_{2} \\
\vdots \\
\underline{\boldsymbol{\mu}}_{N}
\end{array}\right]=\left[\begin{array}{c}
\mathbf{D B}_{1} \mathbf{W}_{1} \\
\mathbf{D B} \mathbf{W}_{2} \\
\vdots \\
\mathbf{D B}_{N} \mathbf{W}_{N}
\end{array}\right] \underline{X}+\left[\begin{array}{c}
\underline{V}_{1} \\
\underline{V}_{2} \\
\vdots \\
\underline{V}_{N}
\end{array}\right]=\mathbf{H} \underline{X}+\underline{V}
$$

From many available estimators, which estimate a HR image from a set of noisy LR images, one may choose to find the most probable $\underline{X}$, given the measurements, $\underline{\mu}$, that is the maximum a-posterior probability(MAP), which can be described as to maximize

$\operatorname{Pr}\{\underline{X} \mid \underline{\mu}\}$

Where $\operatorname{Pr}\{\underline{X} \mid \underline{\mu}\}$ is the probability of the HR image, $\underline{X}$, given the measurements $\underline{\mu}$.

$\operatorname{Pr}\{\underline{X} \mid \underline{\mu}\}=\frac{\operatorname{Pr}\{\underline{\mu} \mid \underline{X}\} \operatorname{Pr}\{\underline{X}\}}{\operatorname{Pr}\{\underline{\mu}\}}$

Therefore, the solution for the maximum a-posteriori probability is described as

$$
\begin{aligned}
\underline{\hat{X}}_{M A P} & =\underset{\underline{X}}{\operatorname{ArgMax} \operatorname{Pr}}\{\underline{X} \mid \underline{\boldsymbol{\mu}}\} \\
& =\underset{\underline{X}}{\operatorname{ArgMax}} \operatorname{Pr}\{\underline{\mu} \mid \underline{X}\} \operatorname{Pr}\{\underline{X}\}
\end{aligned}
$$

By assuming Gaussian distribution for noise and Gibbs distribution with some energy function $\mathrm{A}(\underline{\mathrm{X}})$ for the prior, we get

$$
\begin{aligned}
& \operatorname{Pr}\{\underline{X}\}=\text { Const } \cdot \exp \{-A\{\underline{X}\}\} \\
& \underline{\hat{X}}_{M A P}=\underset{\underline{X}}{\operatorname{ArgMax}} \operatorname{Pr}\{\underline{\boldsymbol{\mu}} \mid \underline{X}\} \operatorname{Pr}\{\underline{X}\} \\
& =\underset{\underline{X}}{\operatorname{ArgMin}} \sum_{i=1}^{N}\left\|\mathbf{D B}_{i} \mathbf{W}_{i} \underline{X}-\underline{\boldsymbol{\mu}}_{i}\right\|^{2}+\lambda A\{\underline{X}\}
\end{aligned}
$$

The last term $\mathrm{A}\{\underline{X}\}$ represents the regularization term and $\lambda$ represents the regularization parameter. Therefore, the resolution enhancement problem can be described as to minimize

$J(\underline{X})=\sum_{i=1}^{N}\left[\left\|\mathbf{Q}_{i} \underline{X}-\underline{\mu}_{i}\right\|_{2}^{2}\right]+\lambda\|\underline{X}\|_{2}^{2}$ 
where $\boldsymbol{Q}_{i}=\boldsymbol{D} \boldsymbol{B}_{i} \boldsymbol{W}_{i}$. In a similar way as in (4), Eq. (12) can be solved recursively, by using adaptive row projection as

$X^{j+1}=X^{j}+\frac{\widehat{\mu}_{i, k}-\left\langle\bar{q}_{i, k}, X^{j}\right\rangle_{2}}{\left\|q_{i, k}\right\|_{2}^{2}} \bar{q}_{i, k}$

where $\mathrm{j}$ denotes the inner iteration, $\hat{\mu}_{i, k}$ is the $\mathrm{k}$-th component of the $\mathrm{i}$-th measurement, $\hat{\mu}_{i}=\boldsymbol{Q}_{i}{ }^{*} \mu_{i}$ and $\bar{q}_{\mathrm{i}, \mathrm{k}}$ is the conjugate complex of $\mathrm{q}_{\mathrm{i}, \mathrm{k}}$ which is the k-th row of $\widehat{\boldsymbol{Q}_{\boldsymbol{l}}}=\boldsymbol{Q}_{i}{ }^{*} \boldsymbol{Q}_{i}+$ $\bar{\lambda} \boldsymbol{I}$.

\subsection{Reconstruction of High-Resolution CT Images}

One problem of performing resolution enhancement in two steps, reconstruction and SR, is that it requires more parameters to be tuned. In addition, the performing the resolution enhancement in two steps is computationally complex. Therefore, rather than performing resolution enhancement in two steps as shown in the previous section, substituting from (5) into (1) leads to

$\underline{U}_{i}=\mathbf{S D B} \mathbf{W}_{i} \underline{X}+\underline{\boldsymbol{\eta}}_{i}+\mathbf{S} \underline{V}_{i}, \quad i=1: N$

$=\mathbf{S D B}_{i} \mathbf{W}_{i} \underline{X}+\underline{E}_{i}, \quad i=1: N$

With

$\underline{E}_{i}=\underline{\boldsymbol{\eta}}_{i}+\mathbf{S} \underline{V}_{i}$

The image reconstruction problem is to minimize

$J(\underline{X})=\sum_{i=1}^{N}\left[\left\|\mathbf{S} \mathbf{D B}_{i} \mathbf{W}_{i} \underline{X}-\underline{U}_{i}\right\|^{2}\right]+\lambda\|\underline{X}\|_{2}^{2}$

$\underline{X}$ is the HR image. Without loss of generality, we assume that the blurring operator is the same for all images, and then the cost function in (16) can be simplified to

$J(\underline{X})=\sum_{i=1}^{N}\left[\left\|\mathbf{A}_{i} \underline{X}-\underline{U}_{i}\right\|_{2}^{2}\right]+\lambda\|\underline{X}\|_{2}^{2}$

With $\mathbf{A}_{\mathrm{i}}=\mathbf{S D B W}_{\mathrm{i}}$, therefore, the HR CT image, $\underline{X}$, can be reconstructed recursively by

$\underline{X}^{j+1}=\underline{X}^{j}+\sum_{i=1}^{N} \frac{u_{i, k}-\left\langle\bar{a}_{i, k}, X^{j}\right\rangle_{2}}{\left\|a_{i, k}\right\|_{2}^{2}} \bar{a}_{i, k}$

where $\mathrm{u}_{\mathrm{i}, \mathrm{k}}$ is the k-th component of $\mathrm{i}$-th measurement, $\widehat{U}_{i}=\boldsymbol{A}_{i}{ }^{*} \underline{U}_{i}$, and $\bar{a}_{\mathrm{i}, \mathrm{k}}$ is the conjugate complex of $\mathrm{a}_{\mathrm{i}, \mathrm{k}}$ which is the k-th row of $\widehat{\boldsymbol{A}}_{i}=\boldsymbol{A}_{i}{ }^{*} \boldsymbol{A}_{i}+\boldsymbol{\lambda} \boldsymbol{I}$. 


\section{Simulation Results \\ 4.1. Experiment Setup}

In this section, we present a set of three experiments illustrating the performance of the proposed algorithm. The experiments are conducted on three simulated phantom sequences, namely, the simulated Phantom-1, simulated Phantom-2 and simulated Phantom-3 images. Each of these sequences contains 4 measured signals, in Sinogram space, to increase resolution by factor $2 \times 2$. The size of each LR image is $60 \times 60$. The relative motions of the generated LR phantom images, with respect to the reference image, are set as $[(0,0),(0,0.5)$, $(0.5,0),(0.5,0.5)]$, where $(\mathrm{x}, \mathrm{y})$ represents shift by $\mathrm{x}$ in $\mathrm{x}$-direction and $\mathrm{y}$ in $\mathrm{y}$-direction. The maximum number of iterations equals 50. Regularization parameter equals $10^{-7}$.

Sinogram space is the data matrix obtained directly from the CT scanner before any kind of processing, which will provide the final reconstructed image. The measured data are computed from the simulated phantom images by measuring the projection of 283 parallel rays for each angle, where 180 angles are used.

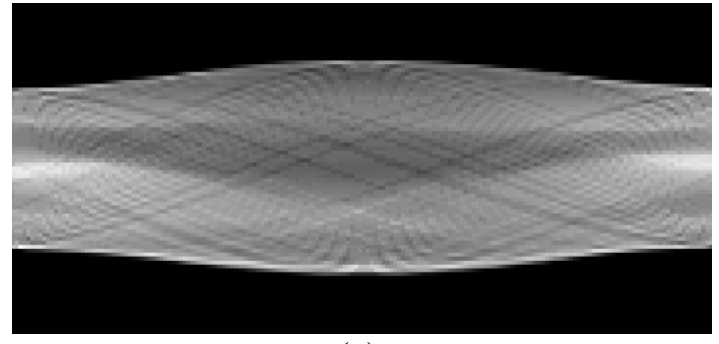

(a)

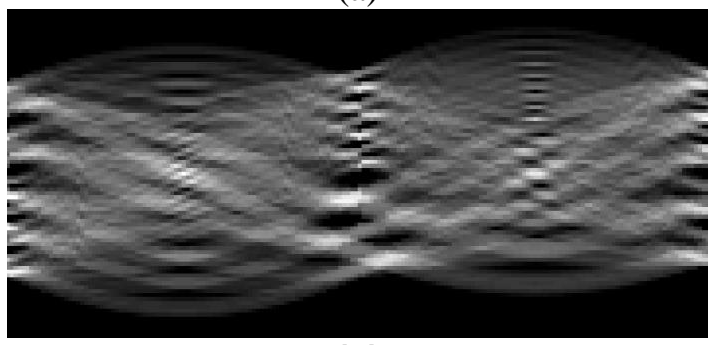

(c)

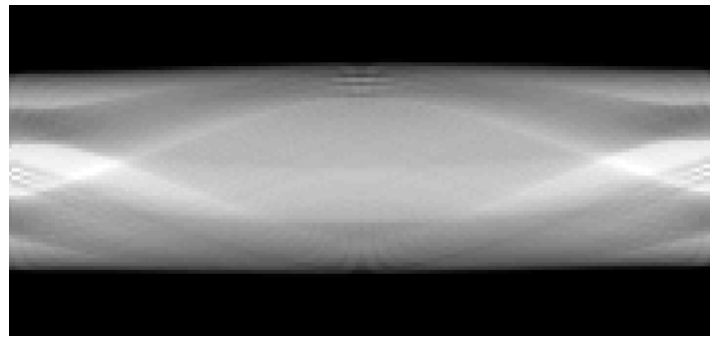

(b)

Figure 1. Measured data in Sinogram space for a), simulated Phantom-1, b) simulated Phantom-2, c) simulated Phantom-3

\subsection{Results and Discussions}

Figures 1a, $1 \mathrm{~b}$ and 1c show images in Sinogram space of simulated phantom-1, simulated phantom-2 and simulated phantom-3 images, respectively.

Figure 2 shows the results of simulated Phantom-1 sequence; in Figure 2a the original simulated phantom image is shown. Figure $2 \mathrm{~b}$ shows one reconstructed LR image. Figures $2 \mathrm{c}$ shows the resolution enhanced reconstructed LR CT image using conventional scheme, i.e. post-process SR, with adopting regularized Kaczmarz minimization. The reconstructed HR CT image using simultaneous resolution enhancement and reconstruction is shown in Figure $2 d$. From Figures $2 b, 2 c$ and $2 d$, we can see that using mult-image resolution enhancement technique as a post-process or using simultaneous resolution enhancement and reconstruction can increase the quality of the reconstructed CT images compared to single LR image reconstruct technique. 
Another example, simulated Phantom-2 sequence, is shown if Figure 3; In Figure 3a the original simulated phantom image is shown. Figure $3 \mathrm{~b}$ shows one reconstructed LR image. Figures 3c shows the resolution enhanced reconstructed LR CT image using conventional scheme, i.e., post-process SR, with adopting regularized Kaczmarz minimization. The reconstructed HR CT image using simultaneous resolution enhancement and reconstruction is shown in Figure 3d. The results in this example confirm the result obtained in Exampe-1, that is resolution enhancement technique reveals more information than that of reconstructed LR images and therefore can enhance the medical diagnosis.

The third example, simulated Phantom-3 sequence, is shown if Figure 4. In Figure 4a the original simulated phantom image is shown. Figure $4 \mathrm{~b}$ shows one reconstructed LR image. Figures $4 \mathrm{c}$ shows the resolution enhanced reconstructed LR CT image using conventional scheme, i.e., post-process SR, with adopting regularized Kaczmarz minimization. The reconstructed HR CT image using simultaneous resolution enhancement and reconstruction is shown in Figure 4d.

The convergence speed of the Kaczmarz method was investigated in many papers [20-21]. It mainly depends on the orthogonality of the system matrix rows. The computational complexity of one Kaczmarz iteration is $O(M N)$, where $\mathrm{M} \times \mathrm{N}$ is dimension of the system matrix, $\mathbf{S}, \mathbf{Q}$ or $\mathbf{A}$ in Eqs. (2), (12) or (17), respectively.

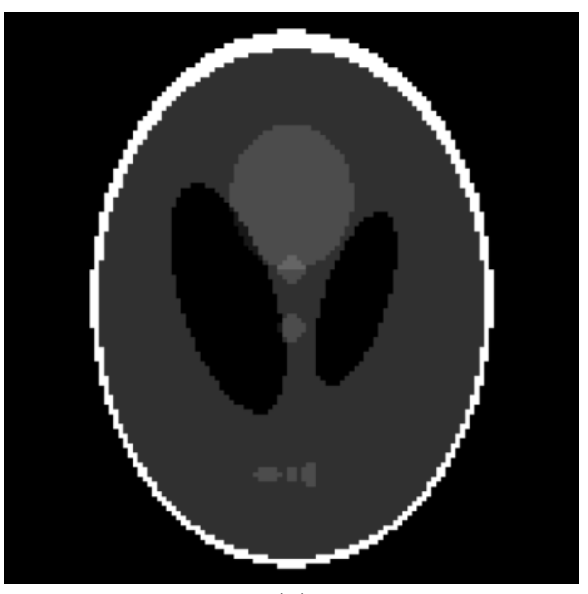

(a)

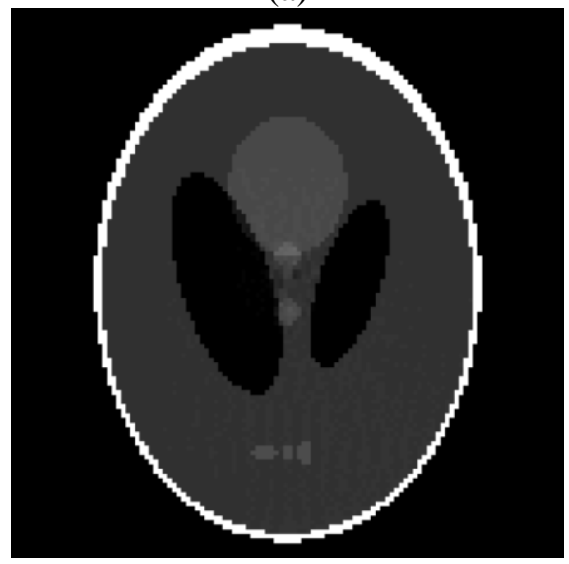

(c)

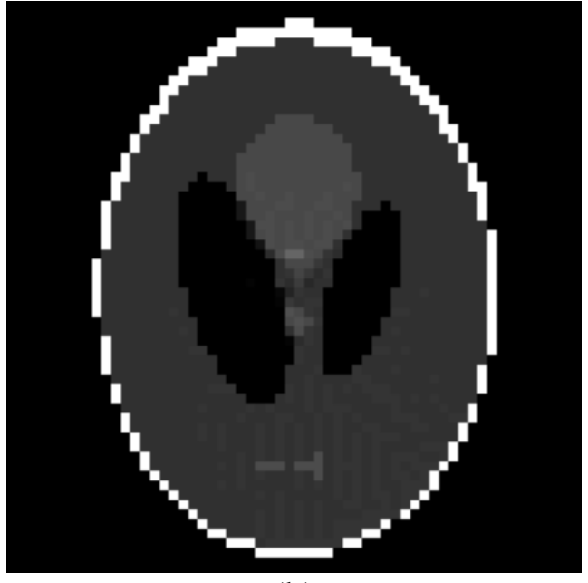

(b)

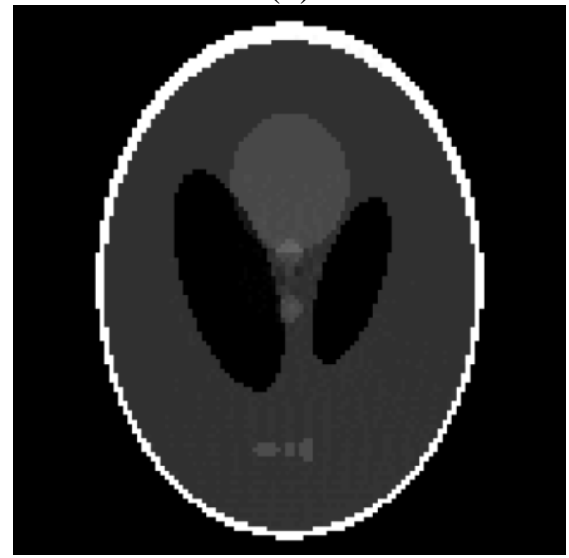

(d)

Figure 2. Example 1, a) Original simulated phantom, b) Reconstructed LR CT image using conventional method, c) Resolution enhanced LR image using 
post-processing SR, d) HR CT using simultaneous reconstruction and resolution enhancement approach

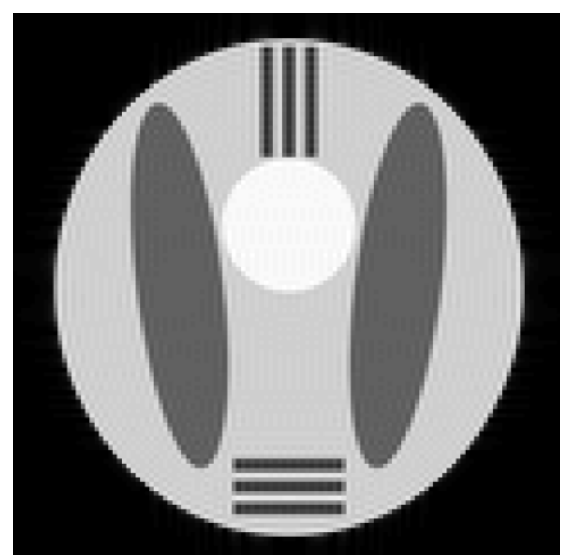

(a)

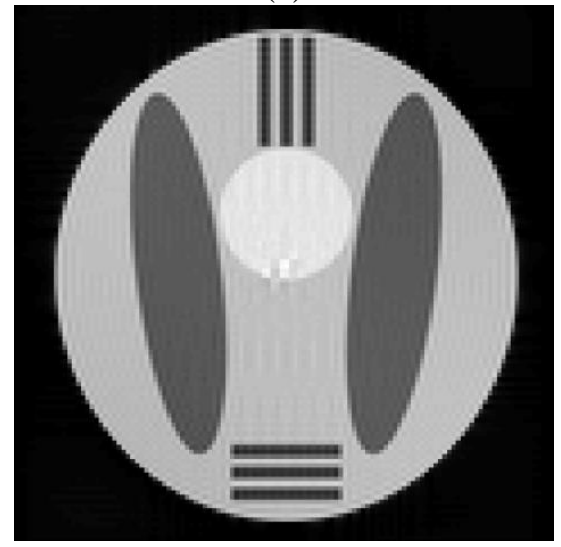

(c)

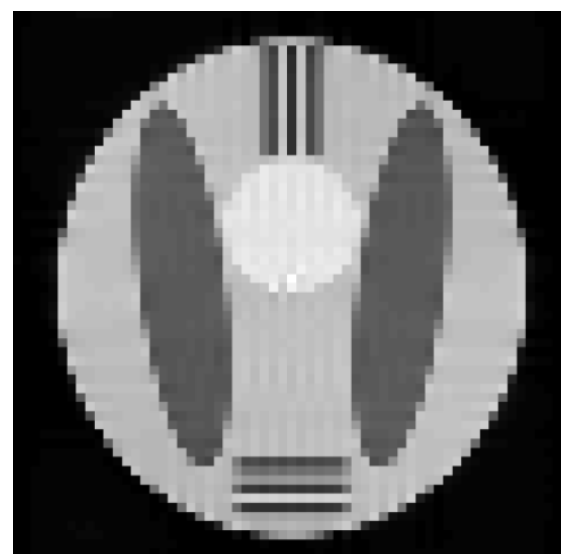

(b)

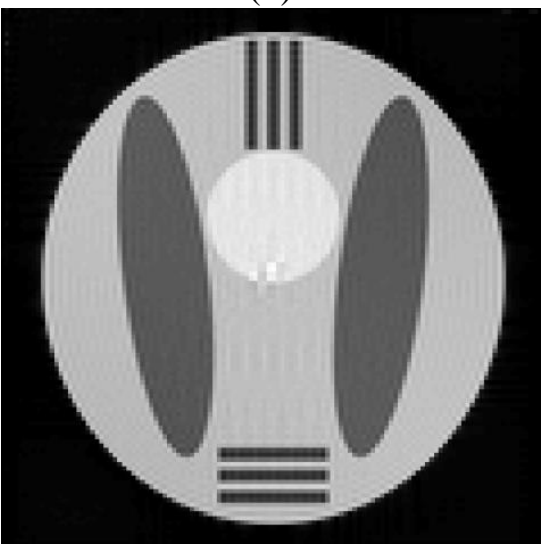

(d)

Figure 3. Example 2, a) Original simulated phantom, b) Reconstructed LR CT image using conventional method, c) Resolution enhanced LR image using post-processing SR, d) HR CT using simultaneous reconstruction and resolution enhancement approach

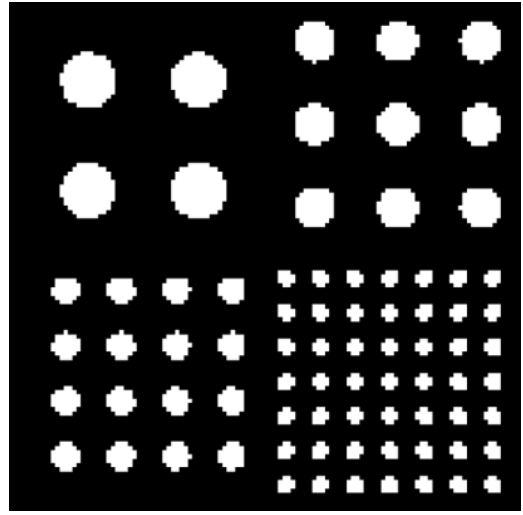

(a)

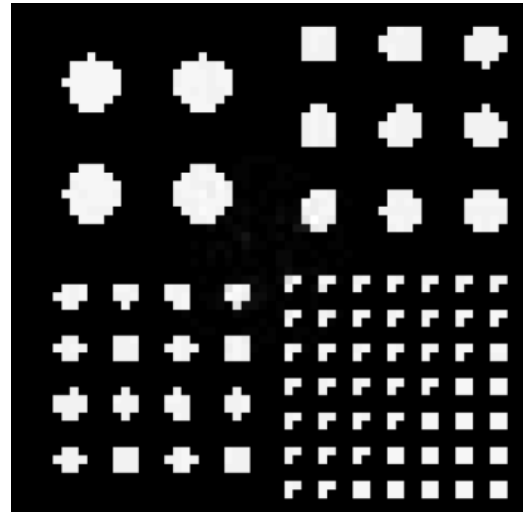

(b) 


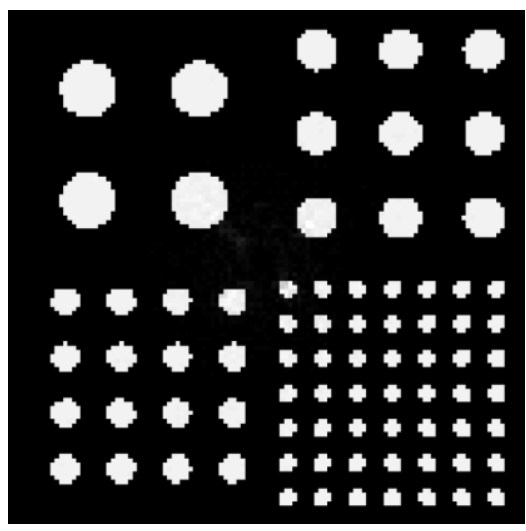

(c)

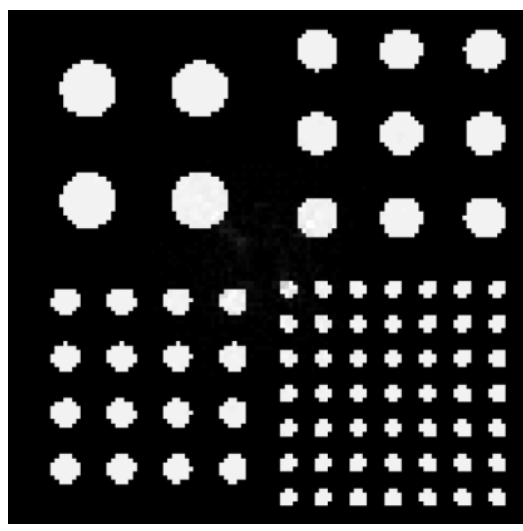

(d)

Figure 4. Example 3, a) Original simulated phantom, b) Reconstructed LR CT image using conventional method, c) Resolution enhanced LR image using post-processing SR, d) HR CT using simultaneous reconstruction and resolution enhancement approach

\section{Conclusions}

We proposed an approach for simultaneous reconstruction and resolution enhancement for CT images. The proposed approach combines multiple measured under-sampled CT signals in Sinogram space, rather than performing resolution enhancement technique as a postprocess. It is proposed to transfer the resolution enhancement problem from pixel domain to the Sinogram domain along with the reconstruction problem. In the proposed scheme, the regularized kaczmarz minimization is adopted.

\section{References}

[1] T. M. Buzug, (Ed.), "Computed tomography from photon statistics to modern cone-beam CT", Springer, (2008).

[2] C. Kak and M. Slaney, "Principles of computerized tomographic imaging", IEEE Press, (1999).

[3] J. Hsieh, B. Nett, Z. Yu, K. Sauer, J. -B. Thibault and C. A. Bouman, "Recent advances in CT image reconstruction", Current Radiology Reports, vol. 1, no. 1, (2013), pp. 39-51.

[4] P. Prakash, M. K. Kalra, J. B. Ackman, D. R. Digumarthy, J. Hsieh, D. Do, J. A. Shepard and M. D. Gilman, "Diffuse lung disease: CT of the chest with adaptive statistical iterative reconstruction technique", Radiology, vol. 256, (2010), pp. 261-269.

[5] D. Marin, R. C. Nelson, S. Y. Schindera, S. Richard, R. S. Youngblood, T. T. Yoshizumi and E. Samei, "Low-tube-voltage, high-tube-current multi-detector abdominal CT: improved image quality and decreased radiation dose with adaptive statistical iterative reconstruction algorithm - initial clinical experience", Radiology, vol. 254, (2010), pp. 145-153.

[6] J. Leipsic, G. Nguyen, J. Brown, D, Sin and J. R. Mayo, "A prospective evaluation of dose reduction and image quality in chest CT using adaptive statistical iterative reconstruction”, AJR Am J Roentgenol, vol. 195, (2010), pp. 1095-1099.

[7] S. Singh, M. K. Kalra, M. D. Gilman, J. Hseigh, H. H. Pien and S. R. Digmarthy, "Adaptive statistical iterative reconstruction technique for radiation dose reduction in chest CT: a pilot study", Radiology, vol. 259, (2011), pp. 565-573.

[8] Y. Ichikawa, K. Kitagawa, N. Nagasawa, S. Murashima and H. Sakuma, "CT of the chest with model-based, fully iterative reconstruction: comparison with adaptive statistical iterative reconstruction", BMC Medical Imaging, (2013), pp. 13-27.

[9] S. -S. Kuo and R. J. Mammone, "Resolution enhancement of tomographic images using the row action projection method", IEEE Transactions on Medical Imaging, vol. 10, no. 4, (1991) December, pp. 593 - 601. 
[10] R. A. Ketcham and W. D. Carlson, "Acquisition, optimization and interpretation of X-ray computed tomographic imagery: Applications to the geosciences", Computers and Geosciences, vol. 27, (2001), pp. $381-400$.

[11] L. G. Villanueva, G. M. Callicó, F. Tobajas, S. López, V. De Armas, J. F. López and R. Sarmiento, "Medical diagnosis improvement through image quality enhancement based on super-resolution", $13^{\text {th }}$ Euromicro Conference on Digital System Design: Architectures, Methods and Tools (DSD), (2010) September 1-3, Lille, France, pp. 259 - 262.

[12] H. Greenspan, "Super-resolution in medical imaging”, The Computer Journal, vol. 52, no. 1, (2009), pp. 4363.

[13] E. Plenge, D. H. J. Poot, M. Bernsen, G. Kotek, G. Houston, P. Wielopolski, L. van der Weerd, W. J. Niessen and E. Meijering, "Super-resolution methods in MRI: can they improve the trade-off between resolution, signal-to-noise ratio, and acquisition time?", Magnetic Resonance in Medicine, vol. 68, (2012), pp. $1983-1993$.

[14] O. A. Omer, "Reconstruction of high-resolution computed tomography image in sonogram space", Journal of Medical and Bioengineering, vol. 3, no. 1, (2014) March.

[15] X. Pan, L. Yu and C. -M. Kao, "Spatial-resolution enhancement in computed tomography", IEEE Transactions on Medical Imaging, vol. 24, no. 2 (2005) February, pp. 246 - 253.

[16] H. M. Shieh, C. Chung and C. L. Byrne, "Resolution enhancement in computerized tomographic imaging", Applied Optics, vol. 47, (2008), pp. 4116-4120.

[17] I. Zhdanov, "The method of augmented regularized normal equations", Computational Mathematics and Mathematical Physics, vol. 52, no. 2, (2012), pp. 194-197.

[18] D. M. S. Kaczmarz, “Angenäherte Auflösung von Systemenlinearer Gleichungen”, English translation: "Approximate Solution for Systems of Linear Equations", Pull. Acad. Polon. Science et Letters, vol. A35, (1937), pp. 355-357.

[19] M. D. Robinson, S. J. Chiu, C. A. Toth, J. A. Izatt, J. Y. Lo and S. Farsiu, "New Applications of Superresolution in medical imaging", Edited P. Milanfar, CRC Press, (2010), pp. 383-412.

[20] L. Elsner, I. Koltracht and P. Lancaster, "Convergence properties of ART and SOR algorithms", Numerische Mathematik, vol. 59, (1991), pp. 91-106.

[21] M. Trummer, "Reconstructing pictures from projections: on the convergence of the ART algorithm with relaxation", Computing, vol. 26, (1981), pp. 189-95. 